\title{
Unsedated esophagogastroduodenoscopy in cirrhotic patients: an impact of topical pharyngeal anesthesia
}

\begin{abstract}
Topical pharyngeal anesthesia is generally used as pretreatment for unsedated esophagogastroduodenoscopy (UEGD). The effectiveness of topical pharyngeal anesthesia for UEGD in cirrhotic patients has been limited reports in the medical literature. The study is aimed to study the impact of topical pharyngeal anesthesia for UEGD procedure in cirrhotic patients in the World Gastroenterology Organization Endoscopy Training Center in Thailand. Retrospectively analyzed the patients on whom UEGD procedures had been performed during the period of December, 2007 to May, 2009 in Siriraj Hospital. The patients' characteristics, duration of procedure, preanesthetic problems and anesthesiarelated adverse events were assessed. The primary outcome variable of the study was the successful completion of the procedure. The secondary outcome variables were anesthesiarelated adverse events and the alteration of hemodynamic parameters. During the study period, there were 346 cirrhotic patients who underwent UEGD procedure. All UEGD procedures were succeeded except four cases. The mean age of the patients was 55.9 \pm 11.9 years, and most were American Society of Anesthesiologists (ASA) class II (53.2\%). The mean duration of procedure was 13.5 \pm 7.4 minutes. Topical pharyngeal anesthesia for UEGD procedure in the cirrhotic patients is relatively safe and effective. Anesthesia-related adverse events in the cirrhotic patients were relatively high. However, these adverse events were mild, transient, without specific interventions, with no adverse sequelae.
\end{abstract}

Keywords: esophagogastroduodenoscopy, unsedated, topical pharyngeal anesthesia, cirrhotic patients, efficacy, adverse event
Volume 3 Issue 4 - 2015

\author{
Somchai Amornyotin, Siriporn Kongphlay \\ Department of Anesthesiology and Siriraj GI Endoscopy Center, \\ Faculty of Medicine Siriraj Hospital, Mahidol University, Thailand
}

Correspondence: Somchai Amornyotin, Department of Anesthesiology and Siriraj GI Endoscopy Center, Faculty of Medicine Siriraj Hospital, Mahidol University, 2, Siriraj, Bangkoknoi, Bangkok 10700, Thailand, Tel +662 419 7990, Fax +662 4II 3256, Email somchai.amo@mahidol.ac.th

Received: October 30,2015 | Published: November 20,2015

\section{Abbreviations:}

UEGD,

esophagogastroduodenoscopy; ASA, american society of anesthesiologists; SD, standard deviation; \%: percentage

\section{Introduction}

Esophagogastroduodenoscopy (EGD) is an endoscopic procedure which is commonly used for diagnostic and therapeutic purposes. The proportion of patients undergoing diagnostic EGD without sedation has been increasing over the past decade. In addition, EGD has several applications specific to the patients with chronic liver disease. ${ }^{1}$ Unsedated esophagogastroduodenoscopy (UEGD) is generally safe and can be well tolerated. ${ }^{2}$ In Thailand, most of diagnostic and screening EGDs are performed without sedation. Topical pharyngeal anesthesia is often used as premedication for EGD procedure. ${ }^{3,4}$ In a meta-analysis, topical pharyngeal anesthesia before EGD with sedation is shown to increase the ease of endoscopy and the patient tolerance. ${ }^{5}$ Topical pharyngeal anesthesia is also often used in UEGD procedure and is thought to improve the patient tolerance. ${ }^{2,6}$ However, the effectiveness of topical pharyngeal anesthesia for UEGD procedure in cirrhotic patients has been limited reports in the medical literature.

\section{Materials and methods}

The study was conducted from December, 2007 to May, 2009 at a large tertiary care referral center, Siriraj Hospital, Bangkok, Thailand. All cirrhotic patients with age at least 18 years of age who presented for diagnostic UEGD were eligible for the study. Exclusion criteria included request intravenous sedation, therapeutic UEGD procedures, and patients with American Society of Anesthesiologists (ASA) physical status of class IV or V. Pharyngeal anesthesia either with topical viscous lidocaine solution or lidocaine spray was performed by the nurse anesthetist or anesthesiology resident supervised by the staff anesthesiologist in the pre-procedure room. Successful completion of the endoscopic procedure was the primary outcome measured. Successful endoscopic procedure was defined as completion of the procedure as intended without additional intravenous sedation. The decision for initiation of intravenous sedation rested on the anesthesiologist performing the procedure. The secondary outcome variables were anesthesia-related adverse events both during and immediately after the procedure as well as the alteration of hemodynamic parameters including systolic and diastolic blood pressure, heart rate and oxygen saturation. These secondary outcome variables were analyzed only in those who successfully completed the unsedated procedure.

The UEGD procedure was performed by either gastroenterology fellow supervised by staff attending physician or by the staff endoscopist. Olympus video esophagogastroduodenoscope (GIF-Q 180, Olympus Corporation, Tokyo, Japan) was used for all EGD procedures. Each patient was monitored in standard manner for noninvasive blood pressure, heart rate, heart rhythm with single lead electrocardiogram, and oxygen saturation with pulse oximetry. No other premedications were administered before the procedure. Anesthesia-related adverse events during and immediately after the procedure were noted. Alteration in hemodynamic parameters was considered as adverse event if any of the following was observed: hypertension or hypotension (increase or decrease in blood pressure by $25 \%$ from baseline), tachycardia or bradycardia (increase or decrease in heart rate by $25 \%$ from baseline), and oxygen desaturation $\left(\mathrm{SpO}_{2}<90 \%\right)$. In addition, other symptoms such as sore throat, nausea, or vomiting were also recorded as adverse event. The results were reported as mean and standard deviation (SD) or percentage (\%) when appropriate. 


\section{Results}

There were 346 cirrhotic patients underwent UEGD procedure during the study period. All UEGD procedures were successfully completed except four cases. These four patients needed additional intravenous sedation. Table 1 shows the characteristics of patients, endoscopy success, duration of procedure and preanesthetic problems. The mean age was $55.9 \pm 11.9$ years. There was slightly more male. The majority of patients were ASA physical status II and III. The preanesthetic problems are also demonstrated in Table 1.

Table I Characteristics of patients, endoscopy success, duration of procedure and preanesthetic problems

\begin{tabular}{ll}
\hline Patient number (n) & 346 \\
\hline Age (yr) (mean, SD) & $55.9(\mathrm{II} .9)$ \\
Gender (n, \%) & \\
Male & $216(62.4)$ \\
Female & $130(37.6)$ \\
Weight (kg) (mean, SD) & $62.4(\mathrm{I} 2.5)$ \\
Height (cm) (mean, SD) & $160.9(8.0)$ \\
Body mass index (kg/m²) (mean, SD) & $24.1(4.2)$ \\
ASA physical status (n, \%) & \\
II & $184(53.2)$ \\
III & $162(46.8)$ \\
Child-Turcotte-Pugh classification (n, \%) & \\
A and B & $329(95.1)$ \\
C & $17(4.9)$ \\
Duration of procedure (min) (mean, SD) & $13.5(7.4)$ \\
Endoscopy success (n, \%) & $342(98.8)$ \\
Preanesthetic problems (n, \%) & \\
Diabetes & $85(24.6)$ \\
Hypertension & $8 \mathrm{I}(23.4)$ \\
Hematologic disease & $12(3.5)$ \\
Cardiovascular disease & $\mathrm{II}(3.2)$ \\
Respiratory disease & $8(2.3)$ \\
Others & $30(8.7)$ \\
\hline
\end{tabular}

The overall anesthesia-related adverse events occurred in 94 patients $(27.2 \%)$. Most of the adverse events are hemodynamic alterations including hypertension (11.6\%), tachycardia (10.7\%), and hypertension and tachycardia (3.2\%). However, these alterations were transient and did not require any specific interventions. Nausea and vomiting occurred in four patients. Additionally, two patients developed sore throat. There were no procedure-related complications (Table 2).

Table 2 Anesthesia-related adverse events during and immediately after procedure $(n, \%)$

\begin{tabular}{ll}
\hline Overall & $\mathbf{9 4}(\mathbf{2 7 . 2 )}$ \\
\hline Hypertension & $40(\mathrm{II} .6)$ \\
Tachycardia & $37(10.7)$ \\
Hypertension and tachycardia & $1 \mathrm{I}(3.2)$ \\
Nausea and vomiting & $4(1.2)$ \\
Sore throat & $2(0.6)$ \\
\hline
\end{tabular}

Table 3 demonstrates the hemodynamic parameters including systolic and diastolic blood pressure, heart rate and oxygen saturation. Mean systolic and diastolic blood pressure was slightly increased at the endoscope insertion and at 5 min after insertion. In addition, mean heart rate increased throughout the procedure when compared with the baseline value. However, mean oxygen saturation during the procedure did not change and was more than $98 \%$.
Table 3 Hemodynamic parameters: systolic and diastolic blood pressure $(\mathrm{mmHg})$, heart rate (beat/minute) and oxygen saturation $\left(\mathrm{SpO}_{2}, \%\right)($ mean, $\mathrm{SD})$

\begin{tabular}{|c|c|}
\hline \multicolumn{2}{|l|}{ Baseline } \\
\hline SBP, DBP & I34.4 (20.7), $75.2(13.7)$ \\
\hline $\mathrm{HR}, \mathrm{SpO}_{2}$ & 74.3 (13.0), 98.7 (I.5) \\
\hline \multicolumn{2}{|c|}{ At Insertion } \\
\hline SBP, DBP & I 42.7 (25.4), $79.8(17.4)$ \\
\hline $\mathrm{HR}, \mathrm{SpO}_{2}$ & $83.2(16.2), 98.6(1.5)$ \\
\hline \multicolumn{2}{|c|}{5 min after insertion } \\
\hline SBP, DBP & I $43.3(24.4), 78.2(16.8)$ \\
\hline $\mathrm{HR}, \mathrm{SpO}_{2}$ & $83.0(16.0), 98.7(1.5)$ \\
\hline \multicolumn{2}{|c|}{10 min after insertion } \\
\hline SBP, DBP & I $38.8(23.6), 77.0(15.8)$ \\
\hline $\mathrm{HR}, \mathrm{SpO}_{2}$ & 80.3 (I5.5), $98.8(1.4)$ \\
\hline \multicolumn{2}{|c|}{15 min after insertion } \\
\hline SBP, DBP & I39.| (23.3), $77.0(15.8)$ \\
\hline $\mathrm{HR}, \mathrm{SpO}_{2}$ & 87.6 (12.2), $98.7(1.5)$ \\
\hline \multicolumn{2}{|c|}{20 min after insertion } \\
\hline SBP, DBP & $141.6(22.0), 76.3(13.9)$ \\
\hline $\mathrm{HR}, \mathrm{SpO}_{2}$ & 82.4 (16.9), 98.6 (I.4) \\
\hline
\end{tabular}

SBP: Systolic Blood Pressure; DBP: Diastolic Blood Pressure; HR: Heart Rate; $\mathrm{SpO}_{2}$ : Oxygen Saturation

\section{Discussion}

UEGD is considered to be safe, feasible, and well tolerated procedure. In addition, the unsedated procedure is well accepted as demonstrated by patients' willingness to repeat the procedure under similar conditions., ${ }^{2,5,7}$ There are a number of advantages associated with performing EGD without sedation. These include a shorter examination time, a decreased incidence of cardiorespiratory adverse events, decreased hospital costs, the ability to work and drive immediately following the procedure. ${ }^{5}$ The tolerance toward unsedated endoscopy is an important factor that determines adequacy and feasibility of the procedure as well as patient and endoscopist acceptance. UEGD in the cirrhotic patients is challenging. Topical pharyngeal anesthesia then becomes important in assisting patient's tolerance to the procedure. The importance and efficacy of pharyngeal anesthesia has been reviewed in a meta-analysis study favoring topical anesthesia in the patients undergoing sedated EGD. ${ }^{5}$ Moreover, several previous studies also confirmed that UEGD in cirrhotic patients appeared to be a safe, well tolerated, and cost-effective procedure. ${ }^{8}$ However, the potential benefit from the use of topical anesthetic preparations before EGD remains controversial. ${ }^{9,10}$ The present study is conducted to determine an impact of topical pharyngeal anesthesia for UEGD procedure in the cirrhotic patients.

The primary outcome of the study was the rate of completion of endoscopy without initiation of intravenous sedation. The result demonstrated that topical pharyngeal anesthesia for UEGD has a good overall successful completion rate $(98.8 \%)$. Our overall success rate in performing UEGD is relatively high. However, our previous study showed that sedated diagnostic EGD procedure was a good technique to improve patient and endoscopist satisfaction as well as increased the comfort and willingness to repeat. ${ }^{11}$ A previous study evaluated the completion rate and determined if sedation could improve the completion rate in 1,539 EGD procedures. The study demonstrated that the completion rate for UEGD procedure was $97.8 \%$. Male patients were more likely to have the EGD without sedation. There were no significant differences in the completion rates of EGD between the patients who received intravenous sedation and topical anesthesia with those who received intravenous sedation alone. ${ }^{12}$ In 
one previous study, the success rate in performing UEGD procedure was $88 \% .{ }^{13}$ Furthermore, a study from England reported the success rate of $92 \% .^{14}$

Several factors associated with successful completion of UEGD procedure have been reported. These included older age, lower level of pre-endoscopic apprehension, smaller endoscope diameter, male gender, and having undergone prior unsedated endoscopy. ${ }^{14,15}$ The higher success rate of completed procedure in this study might be due to two factors. First, the endoscopic procedure is a diagnostic or surveillance UEGD procedure. Second, this study has slightly more male. In our previous study, we evaluated the clinical efficacy of topical viscous lidocaine solution (Group V) and lidocaine spray (Group S) when each is used as a single agent for UEGD procedure in 1,864 adult patients. The procedure was successfully completed in 868 patients $(93.3 \%)$ from group V and 931 patients (99.7\%) from group $\mathrm{S}$. The use of lidocaine spray in UEGD was shown to result in a higher procedural completion rate, greater ease of intubation, and greater patient and endoscopist satisfaction. ${ }^{2}$ The successful completion rate of UEGD procedure in this present study is comparable to the previous report. Our present study used topical viscous lidocaine solution and/ or lidocaine spray for UEGD procedure in cirrhotic patients.

The data regarding the safety of unsedated EGD are limited, and there are no large prospective studies that address safety. In this study, anesthesia-related adverse event rate during and immediately after procedure in the cirrhotic patients were relatively high. These might be due to the definition of adverse events: hypertension or hypotension (increase or decrease in blood pressure by $25 \%$ from baseline), tachycardia or bradycardia (increase or decrease in heart rate by $25 \%$ from baseline), and oxygen desaturation $\left(\mathrm{SpO}_{2}<90 \%\right)$. However, these adverse events were mild, transient, with no adverse sequelae. The result of the present study was similar to the previous result after UEGD procedure in the elderly patients. ${ }^{16}$ The EGD procedure itself has demonstrated to produce various effects on the cardiovascular system. These effects associated endoscopic procedure guide to an increase in sympathetic tone and a decrease in parasympathetic tone causing tachycardia and cardiac arrhythmias. ${ }^{17}$ Pharyngeal anesthesia could be utilized to suppress these effects. However, pharyngeal anesthesia could create some serious adverse events. One single study ${ }^{18}$ suggested that up to $25 \%$ of patients receiving pharyngeal anesthesia demonstrated radiologic evidence of aspiration, a potentially serious adverse event. Although our study did not directly evaluate for aspiration, we did not observed any significant hypoxemia during or after the procedure.

A previous study assessed the oxygen saturation in the patients underwent UEGD procedure by using a pulse oximeter. The study demonstrated that oxygen desaturation is frequently observed during UEGD procedure although severe oxygen desaturation was not common. Furthermore, we cannot predict in which patients desaturation will occur. The study recommended continuous monitoring of arterial oxygenation in all patients during the UEGD procedure. ${ }^{19}$ In addition, Iwao et al. ${ }^{20}$ evaluated the oxygen saturation in the cirrhotic patients underwent UEGD procedure. Oxygen saturation was studied with a pulse oximeter in 80 patients with cirrhosis (44 Child-Pugh's class A, 25 class B, and 11 class C) and in 80 controls. The study showed that oxygen desaturation during UEGD occurred both in cirrhotic patients and in controls. Additionally, the degree of hypoxia and the severity of liver disease failed to show a significant relationship. The study also concluded that a population of patients with cirrhosis did not have an increased risk of oxygen desaturation during UEGD procedure. ${ }^{20}$ Moreover, Banks and colleagues assessed oxygen saturation $\left(\mathrm{SpO}_{2}\right)$ levels in 330 patients underwent routine
UEGD procedures and evaluated the factors related with oxygen desaturation. They concluded that $\mathrm{SpO}_{2}$ levels in the sedated patients were significantly lower than in the unsedated patients. In addition, the $\mathrm{SpO}_{2}$ levels in unsedated patients were not correlated to patient age, gender, duration of procedure, cigarette smoking, endoscope diameter, and basal $\mathrm{SpO}_{2}$ levels. The authors recommended that pulse oximetry was not a prerequisite to performing routine UEGD procedure in the patients without severe systemic disease..$^{21}$ In our study, we used pulse oximetry in all cirrhotic patients.

There are several limitations in this study. First, this study was a retrospective study. Some limitations might be occurred. Second, our study did not assess pre-procedure anxiety and history of previous UEGD procedure which have been shown to be the factors for successful completion of the endoscopic procedures. ${ }^{14}$ Third, the endoscopic procedures were performed by variety of endoscopists including fellows in training. Therefore, the varied experience may have influenced the result including the successful completion rate and alteration of hemodynamic system. Overall, despite these limitations, we are confident, however, that these findings are generalizable to the practice of UEGD procedure in the cirrhotic patients that used topical pharyngeal anesthesia.

\section{Conclusion}

The efficacy of topical pharyngeal anesthesia for UEGD procedure in the cirrhotic patients has been demonstrated. Additionally, the use of topical pharyngeal anesthesia is safe with rare serious adverse events. Although, anesthesia-related adverse events were relatively high, however, these adverse events were mild, transient, without specific interventions. Topical pharyngeal anesthesia may be a good technique for diagnostic UEGD procedure in the cirrhotic patients.

\section{Conflicts of Interest}

The authors do not have any Conflict of interests.

\section{Acknowledgments}

None.

\section{Funding}

None.

\section{References}

1. Early DS, Ben-Menachem T, Decker GA, et al. Appropriate use of GI endoscopy. Gastrointest Endosc. 2012;75(6):1127-1131.

2. Amornyotin S, Srikureja W, Chalayonnavin W, et al. Topical viscous lidocaine solution versus lidocaine spray for pharyngeal anesthesia in unsedated esophagogastroduodenoscopy. Endoscopy. 2009;41(7):581586

3. Amornyotin S, Pranootnarabhal T, Chalayonnavin W, et al. Anesthesia for gastrointestinal endoscopy from 2005-2006 in Siriraj Hospital: a prospective study. Thai J Anesthesiol. 2007;33(2):93-101.

4. Amornyotin S, Prakanrattana U, Chalayonnavin W, et al. Anesthesia for gastrointestinal endoscopy in extremely elderly patients. Thai $J$ Anesthesiol. 2009;35(2):91-99.

5. Evans LT, Saberi S, Kim HM, et al. Pharyngeal anesthesia during sedated EGDs: is "the spray" beneficial? A meta-analysis and systematic review. Gastrointest Endosc. 2006;63(6):761-766.

6. Fisher NC, Bailey S, Gibson JA. A prospective randomized controlled trial of sedation vs. no sedation in outpatient diagnostic endoscopy. Endoscopy. 1998;30(1):21-24 
7. Mulcahy HE, Hennessy E, Connor P, et al. Changing patterns of sedation use for routine out-patient diagnostic gastroscopy between 1989 and 1998. Aliment Pharmacol Ther. 2001;15(2):217-220.

8. Darwin P, Zangara J, Haluszka O, et al. Unsedated esophagoscopy for the diagnosis of esophageal varices in patients with cirrhosis. Endoscopy. 2000;32(12):971-973.

9. Periera SP, Hussaini SH, Hanson PJV, et al. Endoscopy: throat spray or sedation? J R Coll Physicians London. 1994;28(5):411-414.

10. Soma Y, Saito H, Kishibe T, et al. Evaluation of topical pharyngeal anesthesia for upper endoscopy including factors associated with patient tolerance. Gastrointest Endosc. 2001;53(1):14-18.

11. Amornyotin S, Lertakayamanee $\mathrm{N}$, Wongyingsinn $\mathrm{M}$, et al. The effectiveness of intravenous sedation in diagnostic upper gastrointestinal endoscopy. J Med Assoc Thai. 2007;90(2):301-306.

12. Davies JB, Roy SK. Successful completion of upper gastrointestinal endoscopy: a retrospective comparative study on patients who had endoscopy with sedation and without sedation. $J$ Dig Endosc. 2013;4(2):33-38.

13. Wilkins T, Brewster A, Lammers J. Comparison of thin versus standard esophagogastroduodenoscopy. J Fam Pract. 2002;51(7):625-629.

14. Mulcahy HE, Kelly P, Banks MR, et al. Factors associated with tolerance to, and discomfort with, unsedated diagnostic gastroscopy. Scand $J$ Gastroenterol. 2001;36(12):1352-1357.
15. Mulcahy HE, Greaves RR, Ballinger A, et al. A double-blind randomized trial of low-dose versus high-dose topical anesthesia in unsedated upper gastrointestinal endoscopy. Aliment Pharmacol Ther. 1996;10(6):975979

16. Amornyotin S, Kongphlay S. Anesthesia-related adverse event rate and alteration of blood pressure and heart rate during and immediately after unsedated esophagogastroduodenoscopy in elderly patients. $J$ Gastroenterol Hepatol Res. 2015;4(2):1474-1477.

17. Ross C, Frishman WH, Peterson SJ, et al. (2008) Cardiovascular considerations in patients undergoing gastrointestinal endoscopy. Cardiol Rev. 2008;16(2):76-81.

18. Prout BJ, Metreweli C. Pulmonary aspiration after fibre-endoscopy of the upper gastrointestinal tract. Br Med J. 1972;4(5835):269-271.

19. Iwao T, Toyonaga A, Harada $\mathrm{H}$, et al. Arterial oxygen desaturation during nonsedated diagnostic upper gastrointestinal endoscopy. Gastrointest Endosc. 1994;40(3):277-280.

20. Iwao $\mathrm{T}$, Toyonaga A, Harada $\mathrm{H}$, et al. Arterial oxygen desaturation during nonsedated diagnostic upper gastrointestinal endoscopy in patients with cirrhosis. Gastrointest Endosc. 1994;40(3):281-284.

21. Banks MR, Kumar PJ, Mulcahy HE. Pulse oximetry saturation levels during routine unsedated diagnostic upper gastrointestinal endoscopy. Scand J Gastroenterol. 2001;36(1):105-109. 